\title{
Neuronal Migration Dynamics in the Developing Ferret Cortex
}

\author{
Caitlyn C. Gertz ${ }^{1,2,3}$ and Arnold R. Kriegstein ${ }^{1,2}$ \\ ${ }^{1}$ Department of Neurology and ${ }^{2}$ Eli and Edythe Broad Center of Regeneration Medicine and Stem Cell Research, University of California, San Francisco, \\ California 94143, and ${ }^{3}$ Neuroscience Graduate Program, University of California, San Francisco, California 94158
}

During mammalian neocortical development, newborn excitatory and inhibitory neurons must migrate over long distances to reach their final positions within the cortical plate. In the lissencephalic rodent brain, pyramidal neurons are born in the ventricular and subventricular zones of the pallium and migrate along radial glia fibers to reach the appropriate cortical layer. Although much less is known about neuronal migration in species with a gyrencephalic cortex, retroviral studies in the ferret and primate suggest that, unlike the rodent, pyramidal neurons do not follow strict radial pathways and instead can disperse horizontally. However, the means by which pyramidal neurons laterally disperse remain unknown. In this study, we identified a viral labeling technique for visualizing neuronal migration in the ferret, a gyrencephalic carnivore, and found that migration was predominantly radial at early postnatal ages. In contrast, neurons displayed more tortuous migration routes with a decreased frequency of cortical plate-directed migration at later stages of neurogenesis concomitant with the start of brain folding. This was accompanied by neurons migrating sequentially along several different radial glial fibers, suggesting a mode by which pyramidal neurons may laterally disperse in a folded cortex. These findings provide insight into the migratory behavior of neurons in gyrencephalic species and provide a framework for using nonrodent model systems for studying neuronal migration disorders.

Key words: ferret; gyrencephaly; neuronal migration

\section{Significance Statement}

Elucidating neuronal migration dynamics in the gyrencephalic, or folded, cortex is important for understanding neurodevelopmental disorders. Similar to the rodent, we found that neuronal migration was predominantly radial at early postnatal ages in the gyrencephalic ferret cortex. Interestingly, ferret neurons displayed more tortuous migration routes and a decreased frequency of radial migration at later ages coincident with the start of cortical folding. We found that ferret neurons use several different radial glial fibers as migratory guides, including those belonging to the recently described outer radial glia, suggesting a mechanism by which ferret neurons disperse laterally. It is likely that excitatory neurons horizontally disperse in other gyrencephalic mammals, including the primate, suggesting an important modification to the current model deduced primarily from the rodent.

\section{Introduction}

Proper neocortical function, which is crucial for higher-order cognitive processes, relies on precisely regulated patterns of neurogenesis and migration, processes that have been explored intensively in rodent models of cortical development. Excitatory

\footnotetext{
Received June 8, 2015; revised Aug. 31, 2015; accepted Sept. 12, 2015.

Author contributions: C.C.G. and A.R.K. designed research; C.C.G. performed research; C.C.G. analyzed data; C.C.G. and A.R.K. wrote the paper.

This work was supported by Bernard Osher and the National Institute of Neurological Disorders and StrokeNational Institutes of Health (Grant R37 NS35710). We thank William Walantus, Tomasz Nowakowski, and Yingying Wang for technical assistance; Kriegstein laboratory members for helpful discussions and critical reading of the manuscript; and the veterinary staff at UCSF for assistance with ferret handling.

The authors declare no competing financial interests.

Correspondence should be addressed to Arnold R. Kriegstein, Department of Neurology, University of California, 35 Medical Center Way, Box 0525, Room B1038, San Francisco, CA 94143. E-mail: KriegsteinA@stemcell.ucsf.edu. DOI:10.1523/JNEUROSCI.2198-15.2015

Copyright $\odot 2015$ the authors $\quad 0270-6474 / 15 / 3514307-09 \$ 15.00 / 0$
}

pyramidal neurons are descended from radial glia cells (RGs) that reside in the cortical ventricular zone (VZ) and possess fibers that contact both the ventricular and pial surfaces (Malatesta et al., 2000; Miyata et al., 2001; Noctor et al., 2001; Noctor et al., 2002). Most excitatory neurons are generated by intermediate progenitor cells, which are RG daughters that initially reside within the VZ but later collect adjacent to the VZ in the subventricular zone (SVZ) (Haubensak et al., 2004; Noctor et al., 2004). RG fibers have long been known to serve as scaffolds for neuronal migration (Rakic, 1971; Rakic, 1972; Edmondson and Hatten, 1987), with neurons migrating through distinct phases. Newborn pyramidal neurons are initially multipolar (Tabata and Nakajima, 2003; Noctor et al., 2004), but soon become bipolar and migrate radially along $\mathrm{RG}$ fibers, often those belonging to the parent RGs (Noctor et al., 2001), to reach their laminar position within the cortical plate $(\mathrm{CP})$ 
Although the current model of radial migration depicts developmental events in the rodent, much less is known about neuronal migration in the gyrencephalic, or folded, cortex. Differences in the development of a smooth versus folded brain may alter neuronal migration properties and pathways. Specifically, largebrain mammals, including species with a folded cortex, have a greatly expanded SVZ (Smart et al., 2002; Lukaszewicz et al., 2005; Reillo et al., 2011; Martínez-Cerdeño et al., 2012), thereby requiring neurons to travel longer distances to reach the appropriate laminae. In addition, the expanded SVZ contains an increased abundance of progenitors, including outer radial glia (oRGs). oRGs share many features with ventricular RGs (vRGs) but also have distinct properties, including the lack of an apical end foot contacting the ventricular surface (Fietz et al., 2010; Hansen et al., 2010). Importantly, oRG cells display basal fibers that may provide additional guides supporting neuronal migration (Lui et al., 2011). Moreover, the formation of gyri and sulci begins while neurons are still migrating to their terminal positions, thereby increasing the complexity of the migration scaffold.

Evidence suggests that neuronal migration in gyrencephalic species may differ from the model based on the rodent. Retroviral studies in the ferret (Reid et al., 1997; Ware et al., 1999) and primate (Kornack and Rakic, 1995) reveal substantial lateral dispersion of clonally related neurons and tangential orientation of migrating cells has been observed in the developing ferret cortex (O’Rourke et al., 1992; O’Rourke et al., 1995; O'Rourke et al., 1997), suggesting that pyramidal neurons do not follow strict radial paths. However, at the time of these studies, the tangential migration of interneurons was not known (Marín and Rubenstein, 2001). Therefore, the extent of lateral dispersion of pyramidal neurons in the gyrencephalic cortex and the mode by which this may occur remain uncertain. A comprehensive analysis of excitatory neuronal migration in the gyrencephalic cortex may elucidate mechanisms that differ in a smooth versus folded brain and may be important for understanding changes in migration patterns that occur in neuronal migration disorders.

In the current study, we identified a method for visualizing neuronal migration in the ferret, a gyrencephalic carnivore. We found that the majority of neurons migrate radially toward the $\mathrm{CP}$ at ages when the ferret cortex has not yet developed folds. In contrast, later in neurogenesis, when the ferret cortex has just begun folding, neurons migrate in more varied directions and display increased turning along their migratory routes. These more tortuous pathways are accompanied by neurons migrating sequentially along several different vRG and oRG fibers. These behaviors may be characteristic of neurons in a folded cortex and may contribute to the lateral dispersion of pyramidal neurons in gyrencephalic and large-brain mammals.

\section{Materials and Methods}

Cortical slice culture, viral infection, and time-lapse imaging. As described previously (Gertz et al., 2014), embryonic day 27 timed-pregnant ferrets were obtained from Marshall BioResources and maintained according to protocols approved by the University of California San Francisco Institutional Animal Care and Use Committee. Postnatal day 0 (P0) corresponds to the day of birth. P2 or P3 and P6 or P7 ferret kits were deeply anesthetized with isoflurane, decapitated, the brains extracted, and the meninges removed in chilled artificial CSF (ACSF) containing the following (in mM): $125 \mathrm{NaCl}, 2.5 \mathrm{KCl}, 1 \mathrm{MgCl} 2,2 \mathrm{CaCl} 2,1.25 \mathrm{NaH}_{2} \mathrm{PO}_{4}, 25$ $\mathrm{NaHCO} 3$, and $25 \mathrm{D}-(+)$-glucose, bubbled with $95 \% \mathrm{O}_{2} / 5 \% \mathrm{CO}_{2}$. The brain tissue was then embedded in $3.5 \%$ low-melting-point agarose in ACSF and $250-300 \mu \mathrm{m}$ coronal vibratome sections transferred to Millicell-CM slice culture inserts (Millipore) that were immersed in cor- tical slice culture medium [66\% BME, 25\% Hanks, 5\% FBS, 1\% N-2, 1\% penicillin, streptomycin, and glutamine (all Invitrogen) and $0.66 \%$ $\mathrm{D}-(+)$-glucose (zcomSigma-Aldrich)]. An adenovirus (AV-CMV-GFP, $1 \times 10^{10}$; Vector Biolabs) at a dilution of 1:50-1:500 and/or adenoassociated viruses (AAV2-CAG-GFP, $1 \times 10^{13}$, Vector Biolabs; AAV2CAG-tdTomato, $1.6 \times 10^{10}$, Gene Therapy Center Vector Core, University of North Carolina at Chapel Hill) at a dilution of 1:10-1:50 were applied to the slices, which were then cultured at $37^{\circ} \mathrm{C}, 5 \% \mathrm{CO}_{2}, 8 \%$ $\mathrm{O}_{2}$. For quantification of GFP-labeled cells, slices were fixed in $4 \%$ PFA $48-72 \mathrm{~h}$ after viral infection and processed for immunohistochemistry. For time-lapse imaging, cultures were then transferred to an inverted Leica TCS SP5 confocal microscope with an on-stage incubator streaming $5 \% \mathrm{CO}_{2}, 5 \% \mathrm{O}_{2}$, balance $\mathrm{N}_{2}$ into the chamber. Slices were imaged using a $10 \times$ air objective at $15-45 \mathrm{~min}$ intervals for up to $4 \mathrm{~d}$ with repositioning of the $z$-stacks every $12 \mathrm{~h}$.

Ex vivo electroporation. P7 ferret kits were deeply anesthetized with isoflurane, decapitated, the brains extracted, and the meninges removed in chilled ACSF. A micropipette was used to inject 1-2 $\mu \mathrm{l}$ of CMVtdTomato plasmid DNA $(1.5 \mu \mathrm{g})$ into the ventricles of both hemispheres. The positive paddle of the electrode was placed on the lateral cortex and 5 pulses at $52 \mathrm{mV}$ applied (50 ms with $1 \mathrm{~s}$ intervals). Brains were immediately transferred to fresh, chilled ACSF and processed for cortical slice cultures.

Immunohistochemistry, confocal imaging, and cell counts. Cortical slice cultures were fixed in $4 \%$ PFA in PBS overnight and then stored in PBS plus $0.01 \%$ sodium azide at $4^{\circ} \mathrm{C}$. Slices were subjected to boiling citratebased antigen retrieval solution (Vector Laboratories) for $20 \mathrm{~min}$, permeabilized, and placed in blocking buffer (PBS plus $2 \%$ Triton X-100, $10 \%$ serum, and $0.2 \%$ gelatin) for $1 \mathrm{~h}$. Primary antibodies were diluted in blocking buffer and applied to slices for $36-60 \mathrm{~h}$ at $4^{\circ} \mathrm{C}$. Slices were washed with PBS plus $0.5 \%$ Triton X-100 and then incubated in secondary antibodies diluted in blocking buffer for $36 \mathrm{~h}$. Images were acquired on a Leica TCS SP5 X laser confocal microscope. Primary and secondary antibodies used: mouse anti-Satb2 (Santa Cruz Biotechnology, sc-81376, 1:250), rabbit anti-Satb2 (Abcam, ab34735, 1:1000), goat anti-SOX2 (Santa Cruz Biotechnology, sc-17320, 1:250), chicken anti-GFP (Aves Laboratories, GFP-1020, 1:1000), mouse anti-Lhx6 (Santa Cruz Biotechnology, sc-271433, 1:250), Alexa Fluor 488, 546, 594, or 647-conjugated donkey anti-goat, anti-rabbit, anti-mouse IgG (Invitrogen, 1:500), and Alexa Fluor 488 donkey anti-chicken IgY (Jackson ImmunoResearch, 1:500). Imaris software (Bitplane) was used to determine the percentage of GFP-positive cells expressing different markers. For each age and marker, three cortical slices from different kits obtained from different litters were counted in a region spanning the ventricular surface to the pia. The percentage of GFP cells expressing a specific marker was calculated per slice and averaged between slices, with $>800$ GFP cells analyzed per marker.

Analysis of neuronal migration. Eight AAV2-CAG-GFP-labeled imaging fields of frontoparietal lateral cortex from 4 P2/3 nonlittermate ferrets of either sex and 12 imaged positions of the same region from 3 P6/7 nonlittermate ferrets of either sex were analyzed. The location of the imaged fields spanned the entire cortex from the ventricular surface to the pia. Maximum intensity projections of the collected stacks $(5-10 \mu \mathrm{m}$ step size) were compiled, generated into videos, and analyzed using Imaris software. ImarisTrack was used to generate tracks of the position of GFP cells over time. Tracks were then manually edited to correct for software error and the $z$ positions set to a constant value so that only the $x$ and $y$ values of a track changed over time. Track statistics, including track duration, length, average speed, straightness, and displacement, were generated by the software and exported for further analysis. The direction of migration was determined using the $x$ and $y$ displacement values of the last time point of a track (see Fig. 2). According to these values, each track was assigned to a quadrant, with positive $x$ and $y$ values assigned to quadrant I, negative $x$ and positive $y$ to quadrant II, negative $x$ and $y$ to quadrant III, and positive $x$ and negative $y$ to quadrant IV. The $x, y$ coordinates were then converted into polar coordinates using the equation $\tan ^{-1}(y / x)$ and the angle adjusted according to quadrant (no adjustment for tracks in quadrant $\mathrm{I}, 180^{\circ}$ added to tracks in quadrant II and III, and $360^{\circ}$ added to tracks in quadrant IV). The direction of the 
cortical plate was set to $45-135^{\circ}$, with angles adjusted for imaging fields in which the direction of the cortical plate differed from this orientation.

Graphs and statistics. Scatter plot, box plot, and column graphs (mean \pm SEM) were generated in GraphPad Prism software. Displacement graphs were made in Imaris. The ROUT method in Prism was used to determine mean track speed outliers $(Q=1 \%)$ and an unpaired $t$ test with Welch's correction used to assess straightness. Rose plots were generated in Oriana with vector sum $(r)$ indicated with a red arrow. A Watson-Williams $F$ test and Mardia-Watson-Wheeler test were used to assess the mean and distribution of the direction of migration, respectively.

\section{Results \\ AAV2-CAG-GFP labels migrating neurons in the developing ferret cortex}

We have previously used time-lapse imaging to analyze VZ and SVZ progenitor behaviors in the ferret (Gertz et al., 2014) and human cortex (Hansen et al., 2010; LaMonica et al., 2013; Ostrem et al., 2014) by infecting cortical slices with an adenovirus in which GFP is expressed under control of the cytomegalovirus promoter (AV-CMV-GFP). Although this technique robustly labels cortical progenitors, it does not readily label migrating neurons. We found that an adeno-associated virus (serotype 2) with GFP expressed under the control of the cytomegalovirus early enhancer/chicken $\beta$ actin promoter (AAV2-CAG-GFP) preferentially labels postmitotic cells, including migrating neurons (Fig. $1 A$ ). Post-time-lapse fate staining confirmed pyramidal neuron identity because the GFP-positive cells expressed the upper-layer neuronal marker Satb2 and lacked expression of the progenitor marker Sox2 (Fig. 1A).

We characterized neuronal migration in the frontoparietal cortex (Fig. $1 B$ ) at two developmental ages: P2 to P3 (P2/3), when the SVZ is expanded and can be divided into an inner and outer region but the cortex has not yet begun to fold, and P6 to P7 (P6/7), when the cortex has just begun to develop gyri and sulci (Smart and McSherry, 1986a; Smart and McSherry, 1986b; Reillo and Borrell, 2012; Poluch and Juliano, 2015). Whereas migration of excitatory neurons continues past the first postnatal week, only a small number of layer II excitatory neurons are generated in the P2 frontoparietal cortex, with cortical neurogenesis nearing completion in this region by P7 (McConnell, 1988; Jackson et al., 1989; Noctor et al., 1997). Although the majority of labeled cells displayed exclusively migratory behaviors, a small population underwent mitosis and was therefore excluded from our analysis (data not shown). Figure $1 C$ shows migration tracks of GFP-positive cells at $\mathrm{P} 2$.

The migration of individual neurons was analyzed for an average of $27.6 \mathrm{~h}$ and $30.3 \mathrm{~h}$ at $\mathrm{P} 2 / 3(n=277)$ and P6/7 $(n=$ $302)$, respectively. Based on this investigation, we calculated the average migration speed to be $11.9 \mu \mathrm{m} / \mathrm{h}$ at $\mathrm{P} 2 / 3$ and 17.9 $\mu \mathrm{m} / \mathrm{h}$ at $\mathrm{P} 6 / 7$. The vast majority of cells had average migration speeds between 4 and $20 \mu \mathrm{m} / \mathrm{h}$, comparable to the speed of pyramidal neuron migration previously reported in the rodent (Takahashi et al., 1996; Nadarajah et al., 2001) and ferret (O'Rourke et al., 1992; O’Rourke et al., 1997) (Fig. 1D). Cells displayed saltatory locomotion consistent with RG-guided migration (Nadarajah et al., 2001) (Fig. $1 A, E$ ).

A minor population of fast-migrating neurons, with an average migration speed of $49.3 \mu \mathrm{m} / \mathrm{h}$ and ranging up to 105 $\mu \mathrm{m} / \mathrm{h}$, became more prevalent at P6/7 (14.9\%) compared with P2/3 (1.8\%) (Fig. 1D). These cells were identified as statistical outliers and are depicted as red data points in Figure $1 D$. Because this average speed is similar to migrating ferret neu- rons leaving the early postnatal ganglionic eminence (Abbah and Juliano, 2014) and because rodent inhibitory interneurons have been shown to migrate substantially faster than pyramidal neurons (Ang et al., 2003; Valiente and Martini, 2009), we hypothesized that these fast-migrating cells are interneurons originating from the ventral forebrain. Due to their rapid migration speeds, we were unable to locate and assess marker expression after time-lapse imaging. However, we determined the proportions of virally-labeled medial ganglionic eminence-derived cortical interneurons and excitatory pyramidal neurons by staining AAV2-CAG-GFP-infected slices with antibodies against Lhx6 (Cobos et al., 2006; Fogarty et al., 2007) and Satb2, respectively (Fig. 1G). At P2/3 and P6/7, only $2-4 \%$ of GFP-positive cells expressed Lhx6, compared with $20-30 \%$ that expressed Satb2 (Fig. 1F). Because Lhx6 is not expressed by cortical interneurons derived from the caudal ganglionic eminence (Butt et al., 2005; Miyoshi et al., 2010), these percentages may be an underestimate of the number of cortical interneurons labeled by the virus. These results suggest that the minority population of fast-migrating cells is likely interneurons and were therefore excluded from subsequent analyses.

\section{Cortical-plate-directed migration of ferret neurons decreases with age}

To determine the directionality of migrating neurons in the developing ferret cortex, we assessed track displacement over time and plotted migration paths along the $x$ - and $y$-axis from the same point of origin (Fig. 2A). We found that the majority of migration tracks in the VZ/SVZ at $\mathrm{P} 2 / 3$ and in the intermediate zone (IZ)/CP at $\mathrm{P} 6 / 7$ were in the direction of the $\mathrm{CP}$, whereas the direction of $\mathrm{P} 6 / 7$ migration tracks in the VZ/SVZ were more varied. To measure this quantitatively, the angle of migration of each cell was calculated based on the $x, y$ displacement values from the last track time point, with the direction of the CP defined as $45-135^{\circ}$. Although the mean angle of migration direction was not significantly different between all analyzed P2/3 $\left(92.71^{\circ}, n=272\right)$ and P6/7 $\left(97.21^{\circ}, n=257\right)$ neurons, the length of the vector became noticeably shorter at P6/7 $(r=0.39)$ compared with $\mathrm{P} 2 / 3(r=0.71)$ (Fig. $2 B)$. In addition, the distribution of migration angles of all neurons was significantly different between P2/3 and P6/7 ( $p=4.08 \times$ $\left.10^{-7}\right)$, with a greater frequency of cells migrating in nonradial directions at $\mathrm{P} 6 / 7$. This age-related difference in the distribution of migration direction was more pronounced for neurons migrating through the germinal zones (VZ/SVZ; $p=8.57 \times$ $\left.10^{-8}\right)$ than through the IZ/CP $(p=0.02)$, with the vector length longer in the P6/7 IZ/CP $(r=0.52)$ compared with the P6/7 VZ/SVZ ( $r=0.36$; Fig. $2 B)$.

These data suggest that, at early stages of ferret cortical development, when the cortex is not yet folded, the majority of neurons migrate radially toward the $\mathrm{CP}$, similar to the rodent. However, later in neurogenesis, when the cortex has just started to fold, neurons do not follow strict radial pathways and instead can migrate toward the VZ surface as well as horizontally, particularly when traversing the germinal zones.

\section{Neuronal migration routes become more tortuous at later ages}

In addition to an increase in the frequency of non-CP-directed migration, neurons exhibited more frequent turns at later ages. We quantified this property using a straightness index. Straightness is defined as the ratio of the distance between the 

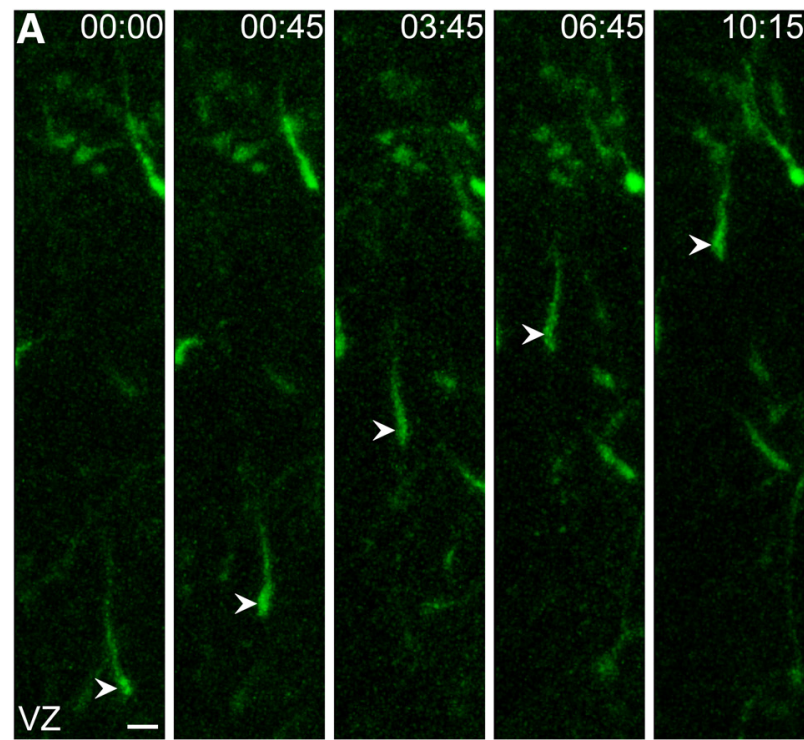

B
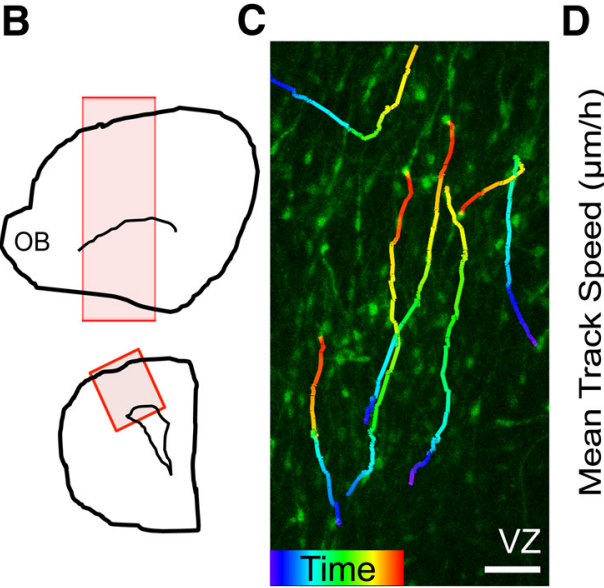

D 120

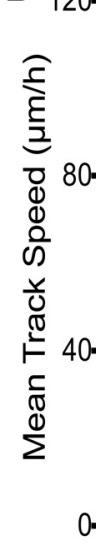

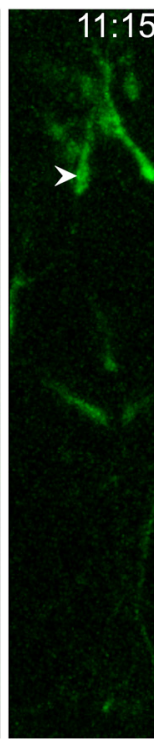
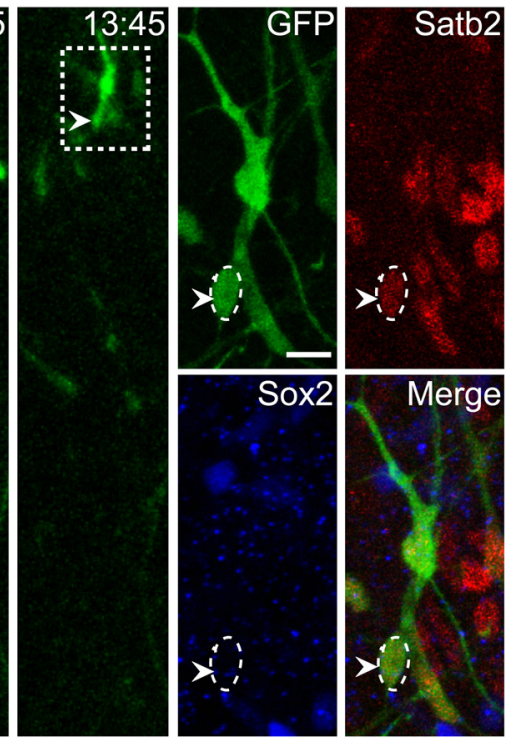

E

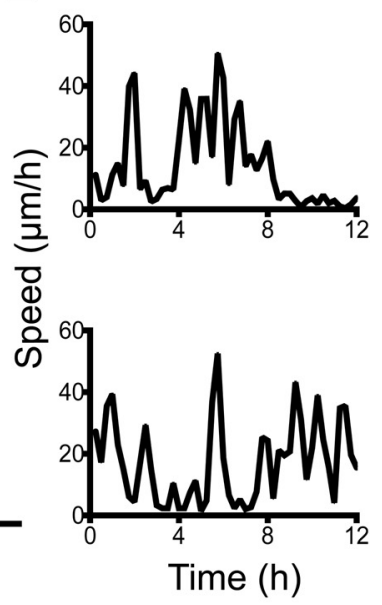

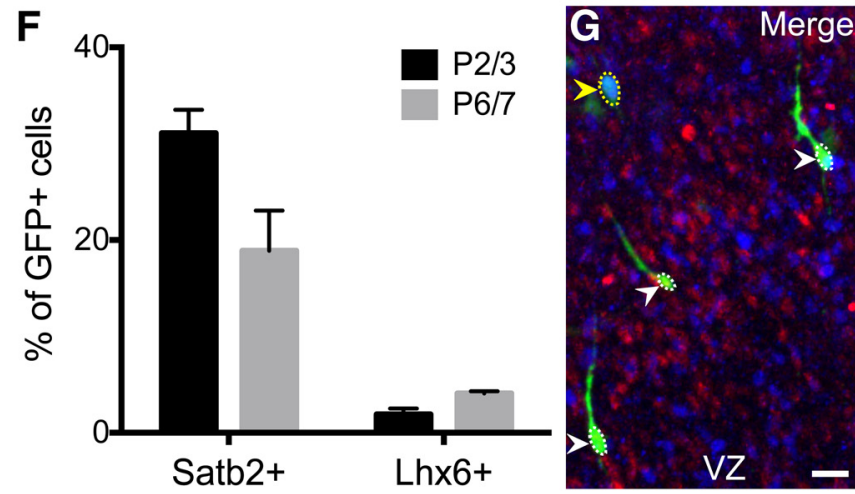
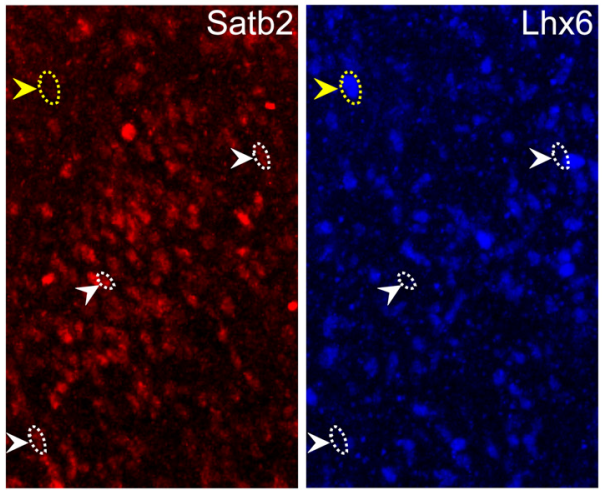

Figure 1. AAV2-CAG-GFP labels migrating neurons in the developing ferret cortex. A, Time-lapse series of a migrating neuron (white arrowhead) labeled with AAV2-CAG-GFP in the P2 ferret cortex. Time elapsed from first frame in series indicated in top right of image (h:min). VZ surface is down. Post-time-lapse fate staining reveals that the migrating GFP cell expresses Satb2 and not Sox2. Scale bars, $20 \mu \mathrm{m}$. B. Schematic of the lateral surface of a P2/3 ferret brain illustrating the frontoparietal location of cultured cortical slices (red box), as well as location of imaging fields in coronal slices (red box: lateral cortex from VZ to pial surfaces). C, Example of a part of an imaging field from a P2 ferret cortical slice labeled with AAV2-CAG-GFP. The migration tracks of GFP cells were generated in Imaris and are shown with a time index indicating the position of the cell over time during the imaging session. VZ surface is down. Scale bar, $50 \mu \mathrm{m}$. D, Scatter plot showing median and interquartile range of the mean track speed of all analyzed cells at P2/3 ( $n=277)$ and P6/7 ( $n=302)$. Cells identified as fast-migrating outliers by the statistical ROUT method are shown in red. $\boldsymbol{E}$, Graphs of migration speed over time of $\mathrm{P} 2$ migrating neurons with average track speeds between 14 and $18 \mu \mathrm{m} / \mathrm{h}$. $\boldsymbol{F}$, Percentage of AAV2-CAG-GFP-labeled cells that express the upper-layer pyramidal neuron marker Satb2 or medial ganglionic eminence-derived interneuron marker Lhx6 (mean \pm SEM). G, Representative image of the outer SVZ from a P7 ferret cortical slice labeled with AAV2-CAG-GFP and stained for Satb2 (red) and Lhx6 (blue). White arrowheads indicate GFP-positive cells expressing Satb2 and not Lhx6; yellow arrowhead indicates a cell expressing Lhx6 and not Satb2. VZ surface is down. Scale bar, $20 \mu \mathrm{m}$. 

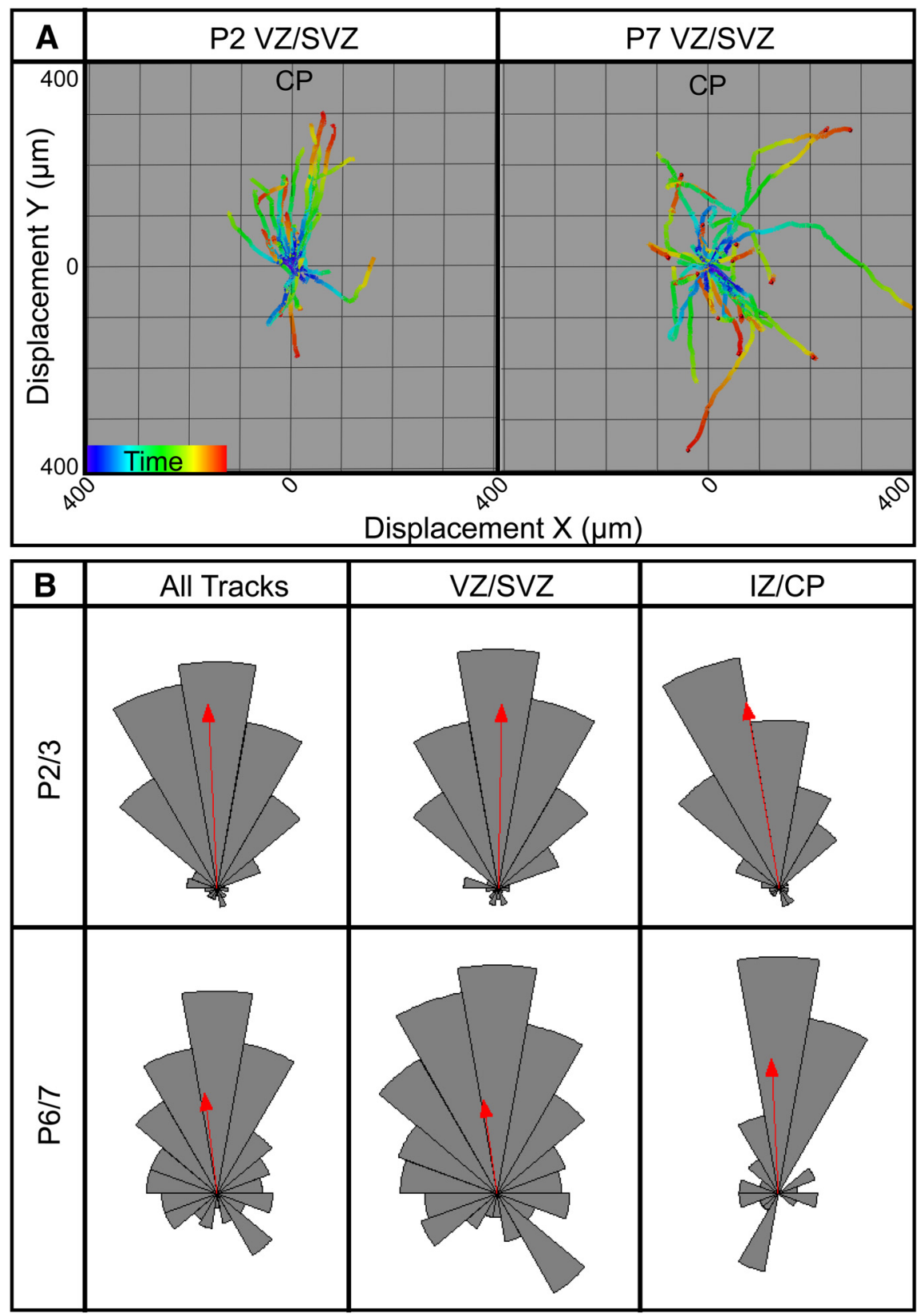

Figure 2. Cortical plate-directed radial migration of ferret neurons decreases with age. $A$, Neuronal migration tracks from a P2 (left) and P7 (right) imaging position in the VZ/SVZ plotted from a common origin as displacement of $x$ and $y$ over time. The direction of the $C P$ is indicated and tracks are shown with a time index indicating the position of the cell over time during the imaging session. $\boldsymbol{B}$, Rose plots of the direction of migration of $\mathrm{P} 2 / 3$ and P6/7 tracks from all imaged fields (minus fast-migrating outliers), VZ/SVZ imaging fields, and IZ/CP imaging fields (left to right). Each wedge represents a $20^{\circ}$ interval with the frequency depicted by the radius length. The direction of the $\mathrm{CP}$ is between $45^{\circ}$ and $135^{\circ}$, with $0^{\circ}$ to the right. The red arrow indicates the vector sum $(r)$ or the weighted average of migration direction.

first and last positions of a track and the actual length of the track (Fig. 3D). We found that the straightness index was significantly decreased at $\mathrm{P} 6 / 7$ compared with $\mathrm{P} 2 / 3$ (Fig. 3A). When average straightness was compared between regions, this age-related decrease was specific to neurons migrating within germinal regions (VZ/SVZ; Fig. 3B). These data suggest that ferret neurons migrating through the VZ and SVZ meander more along their paths at later ages. An example of a P7 neuron migrating within the SVZ and exhibiting several directional turns is shown in Figure $3 C$. The leading process is highly dynamic and, before each turn, appears to retract and then extend in a new direction before the nucleus begins to move.

\section{Ferret neurons can migrate successively along several RG fibers}

The observations that P6/7 ferret neurons migrate in nonradial orientations (Fig. 2) and change directions along their paths (Fig. 3) suggest that neurons may migrate along several different RG fibers on their way to the $\mathrm{CP}$. We used two different techniques to simultaneously image RG fibers and migrating neurons to test this hypothesis. First, AV-CMV-GFP and AAV2-CAG-tdTomato were applied to slices to label ferret RG cells (Gertz et al., 2014) and migrating neurons, respectively. In the P7 SVZ, we observed tdTomatopositive neurons migrating along GFPpositive cell fibers extending toward the pial surface. An example is shown in Figure 4A, where a neuron can be seen migrating along the fiber of an oRG cell. oRG identity was established when the cell underwent mitotic somal translocation characteristic of oRGs (Hansen et al., 2010; Gertz et al., 2014). This observation, though captured in only a few instances, confirms that oRG cells can serve as scaffolds for neuronal migration, a feature that had only been hypothesized previously (Lui et al., 2011; Borrell and Reillo, 2012).

We also labeled RG cells and their fibers by ex vivo electroporation of a CMVtdTomato plasmid, followed by infection of cortical slice cultures with AAV2-CAGGFP to label migrating neurons (Fig. $4 B$ ). Figure $4 C$ shows an example of a GFPpositive neuron that begins migrating toward the $\mathrm{CP}$ along a tdTomato-positive RG fiber. A schematic of the migration path and the RG fibers along which the neuron migrated are shown in Figure $4 D$. In this example, the neuron makes two $180^{\circ}$ directional turns, each of which is preceded by reorientation of the leading process, which contacts and adheres to a different, nearby RG fiber. We observed several instances of neurons switching fibers. These data suggest that ferret neurons that meander along their path and make directional turns can do so by migrating along several different RG fibers, including those belonging to both vRG and oRG cells.

\section{Discussion}

Although recent advances have been made in classifying progenitor behaviors by direct observation in gyrencephalic species (Hansen et al., 2010; Betizeau et al., 2013; LaMonica et al., 2013; Gertz et al., 2014; Ostrem et al., 2014), few observations of neuronal migration have been made in the gyrencephalic cortex. In this study, we identified a method for visualizing cell migration in the gyrencephalic ferret cortex and analyzed pyramidal neuron 
A
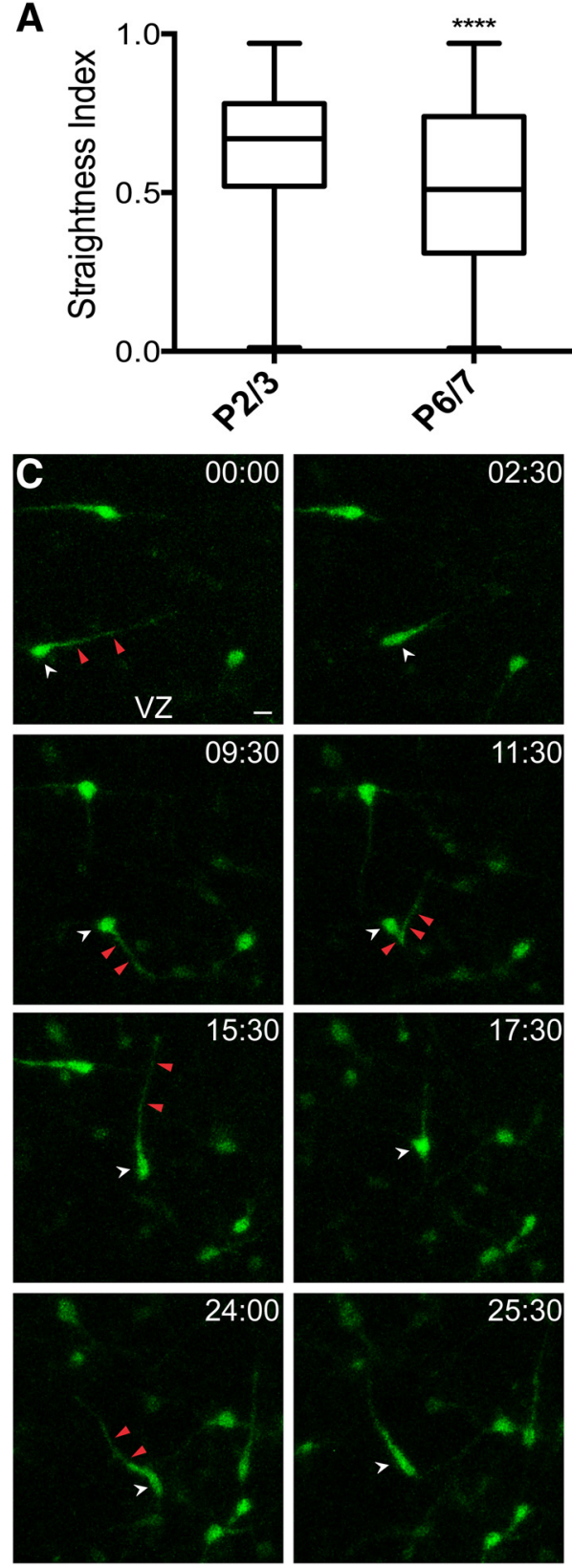
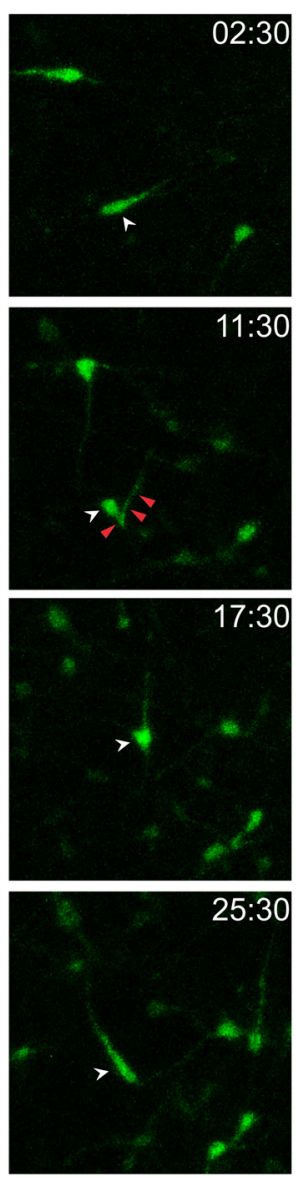

B
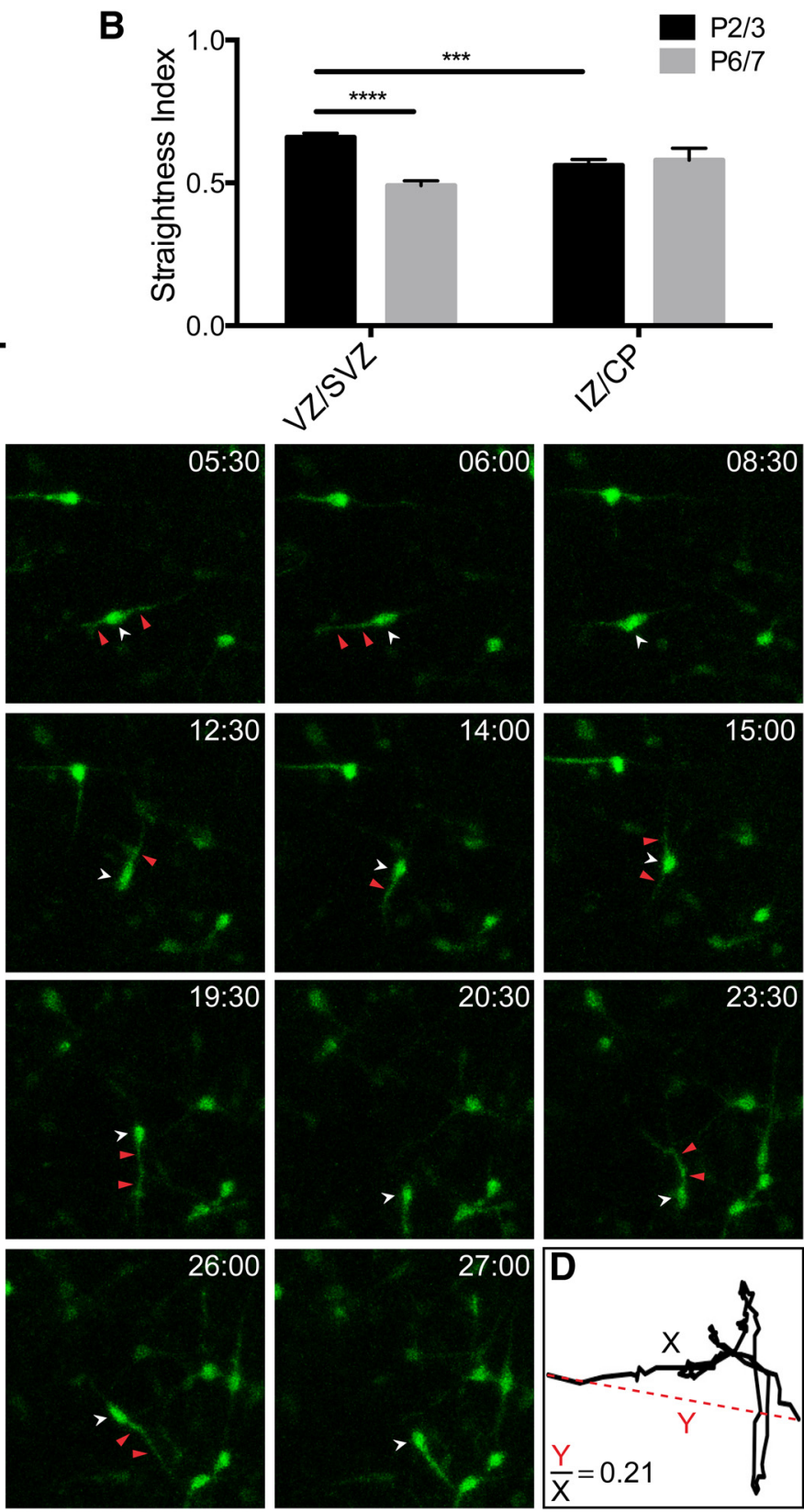

Figure 3. Migration pathways of ferret neurons become more tortuous with age. $A$, Box plot showing minimum and maximum straightness index of all tracks (minus fast-migrating outliers) at P2/3 and P6/7. B, Average track straightness index of migrating neurons according to age and region (mean \pm SEM). C, Time-lapse series of a P7 AAV2-CAG-GFP-labeled neuron in the outer SVZ (white arrowhead) turning directions multiple times during imaging. Directional turns were preceded by reorientation of the leading process (closed red arrowheads). Time elapsed from first frame in series indicated in top right of image ( $\mathrm{h}$ :min). VZ surface is down. Scale bar, $10 \mu \mathrm{m}$. D, Schematic of the migration track of the neuron shown in $\boldsymbol{C}$ (black line). Track straightness is determined by dividing the distance between the first and last points of the track (red dotted line, $Y$ ) by the actual track length (black line, $X$ ), which in this example is $0.21 .{ }^{* * * *} p<0.0001 ;{ }^{* * *} p<0.001$.

migration properties. We observed differences in the migration dynamics of neurons as a function of age, with nonradial, meandering migration more apparent at later ages. This was accompanied by neurons migrating along oRG fibers and switching between different RG fibers along their migration path. This reveals a mode by which late-born neurons can disperse laterally in the developing ferret cortex.

We were able to visualize robust neuronal migration in the ferret by infecting cortical slices with AAV2-CAG-GFP. In contrast, infection of cortical slices with AV-CMV-GFP results in the labeling of few migrating cells (Gertz et al., 2014). Variations in cell infectivity or promoter expression may explain this difference. The viruses may infect different cell populations due to the difference in the viral backbones and serotypes. Alternatively, they may infect similar populations, but GFP levels may vary due to differences in the promoter driving reporter expression. Although CMV and CAG are constitutive promoters commonly used to drive reporter expression in mammalian tissue, differences in expression kinetics and cell-type specificity have been reported (Qin et al., 2010). For example, the CMV promoter can become silenced in specific neuronal subtypes (Gray et al., 2011).

Time-lapse imaging revealed that the majority of AAV2CAG-GFP-labeled cells had migration speeds between 4 and 20 $\mu \mathrm{m} / \mathrm{h}$ and underwent saltatory migration, with periods of movement interspersed with pauses. These features are typical of excitatory neuron migration in the rodent (Nadarajah et al., 

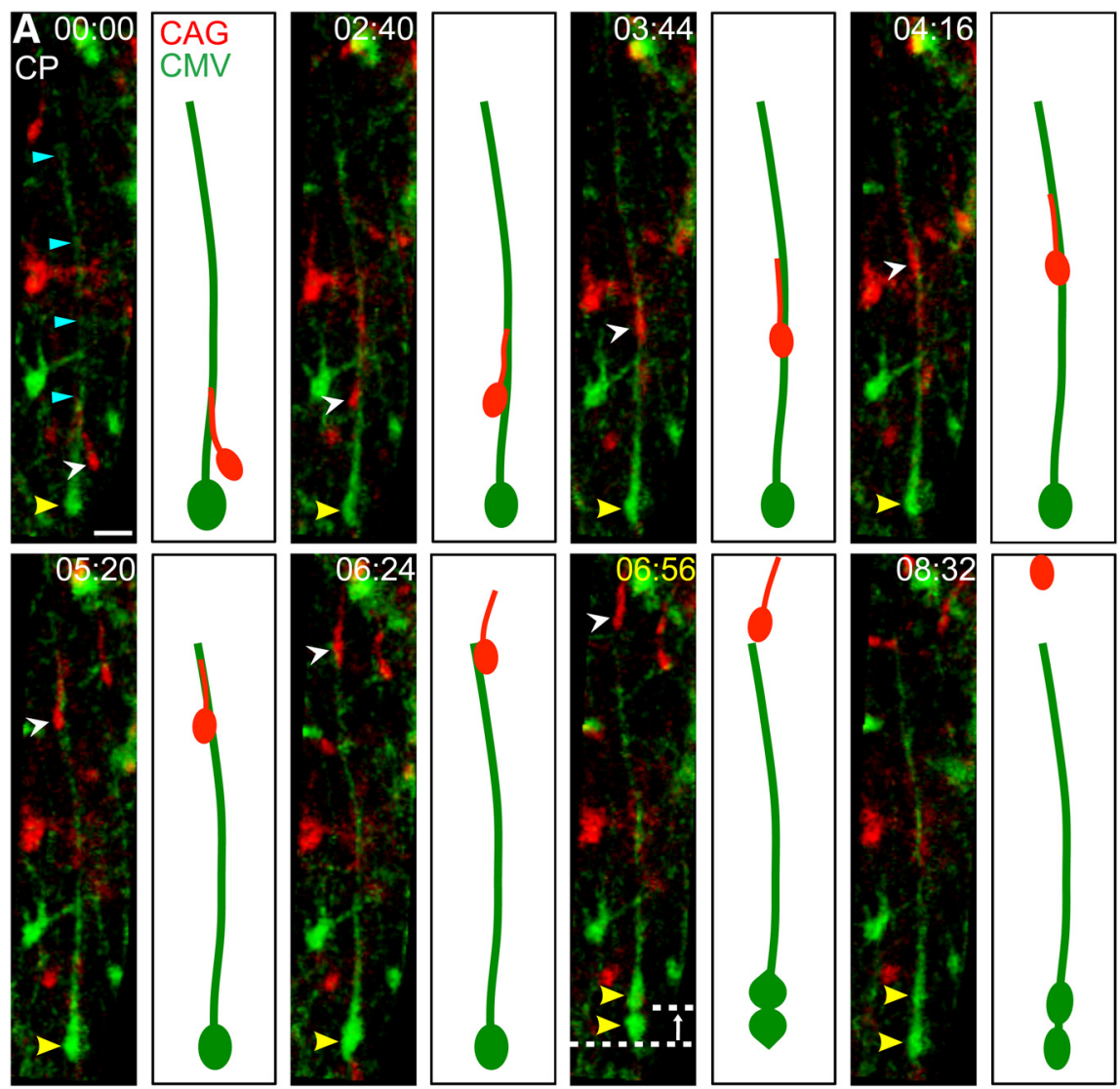

B
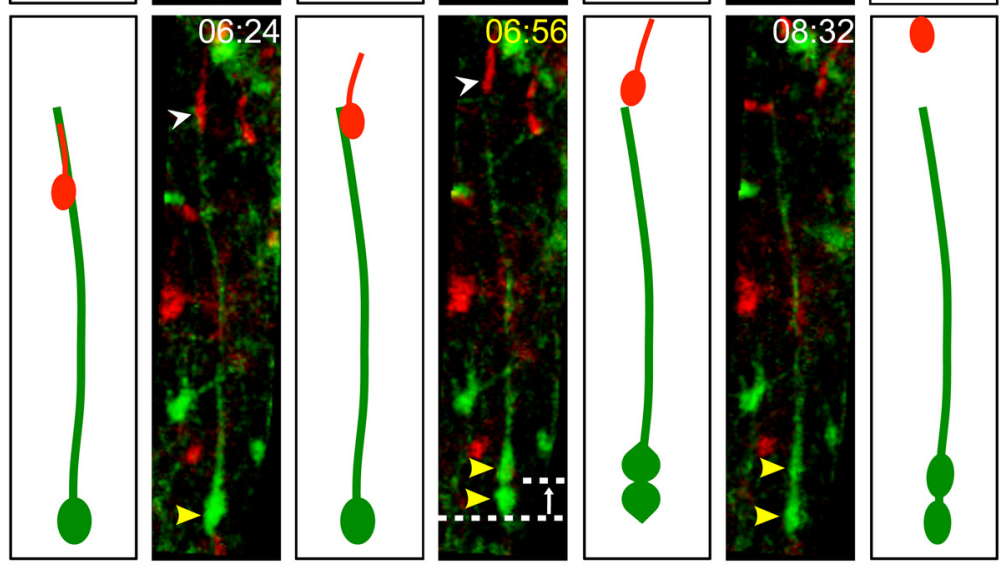

CMV-tdtom
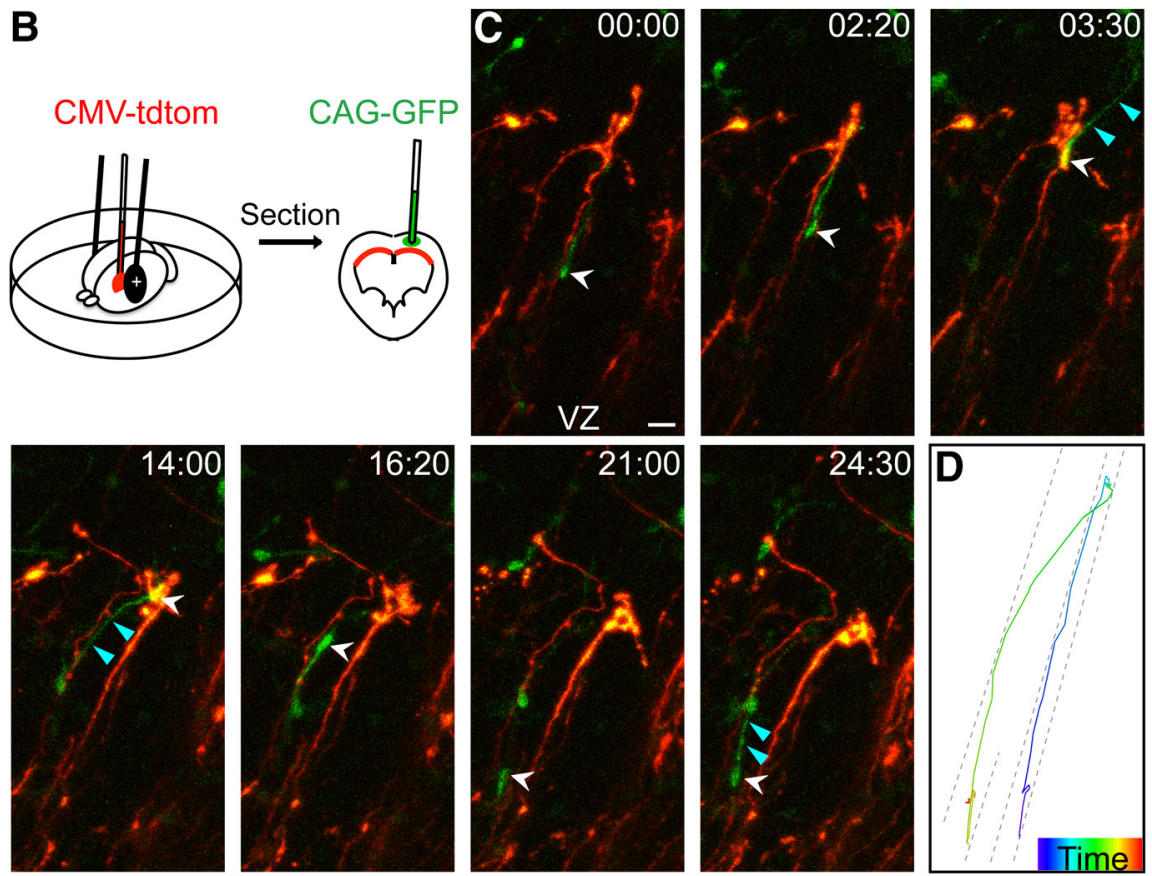

Figure 4. Ferret neurons can migrate promiscuously along different $v R G$ and $0 R G$ fibers. $\boldsymbol{A}$, Time-lapse series of an AAV2-CAGtdTomato-labeled neuron (white arrowhead) migrating along the fiber (light blue arrowheads) of an AV-CMV-GFP-labeled oRG cell (yellow arrowhead) in the P7 OSVZ. Schematics of the time-lapse stills are shown to the right. Time elapsed from first frame in series indicated in top right of image ( $\mathrm{h}: \mathrm{min}$ ) with yellow indicating the time of the mitotic somal translocation division. VZ surface is down. $\boldsymbol{B}$, Schematic of experimental design used in $\boldsymbol{C}$. CMV-tdTomato plasmid DNA was introduced into P7 vRGs in the lateral cortex by ex vivo microinjection into the ventricle followed by electroporation. Coronal vibratome sections were then made and AAV2-CAG-GFP applied to sections via micropipette. C, Time-lapse series of a GFP-positive neuron in the SVZ (white arrowhead) migrating along tdTomato-positive RG fibers. Directional turns were preceded by reorientation of the leading process (light blue arrowheads). Time elapsed from first frame in series indicated in top right of image (h:min). VZ surface is down. D, Migration track of the neuron shown in $C$ with a time index. Gray dotted lines indicate tdTomato-positive RG fibers. Scale bars, $20 \mu \mathrm{m}$.
2001). Migrating ferret neurons exhibited either unipolar or bipolar morphology and possessed a short leading process. Multipolar migration, which has been described at early stages of neuronal migration in the rodent (Tabata and Nakajima, 2003; Noctor et al., 2004; Tabata et al., 2009), was not observed. In addition, we did not find GFP-positive cells with a process extending to the pial surface or displaying somal translocation, features that have been described for cells in the developing ferret cortex (Borrell et al., 2006). It is possible that these morphologies are not adopted by migrating ferret pyramidal neurons or that CAG-GFP is not expressed in multipolar or translocating cells.

At early postnatal ages, the pattern of neuronal migration appeared nearly indistinguishable from the rodent. In general, neurons migrated radially toward the CP. However, later in neurogenesis and shortly after the cortex begins to fold, many neurons migrated through the VZ and SVZ in nonradial directions. These data are consistent with the increased dispersion of clonally related ferret neurons when retrovirus is administered at later developmental ages (Reid et al., 1997; Ware et al., 1999). Adding to diversity in the direction of migration, neurons at later ages frequently changed orientation, sometimes undergoing $180^{\circ}$ turns and retracing their paths. The dynamics of the leading process during these directional changes is reminiscent of rodent interneurons (Valiente and Martini, 2009). Specifically, the leading process undergoes repeated cycles of retraction, extension, and reorientation (Martini et al., 2009). A recent study documented similar exploratory behaviors by ferret neurons leaving the cortical VZ during early postnatal development (Abbah and Juliano, 2014). Upper-layer pyramidal neurons in the developing ferret cortex therefore appear to behave more dynamically than those in the rodent cortex and can migrate both radially and laterally during the bipolar phase of migration.

Changes in the radial fiber scaffold may contribute to neuronal migration differences observed during development. At P2, ferret RG fibers in the SVZ appear parallel and predominantly radial. In contrast, P6 RG fibers undulate and are less radially aligned in the SVZ, becoming more radial in the IZ and CP (Reillo and Borrell, 2012). This could be due in part to oRG cells in the SVZ that have primary fibers that vary in orientation and are not always anchored to the pial surface (Gertz et al., 2014). Because cortical neurons use 
RG fibers as guides for migration, the increasingly nonradial organization of the fiber scaffold over time may cause neurons to display less radial and more varied migration paths at later ages. At P6/7, there was less CP-directed migration by neurons migrating through the VZ/ SVZ compared with cells migrating through the IZ/CP (Fig. 2B), consistent with the patterning of the RG scaffold. As proposed previously (Reillo et al., 2011), a divergent, fanned array of RG fibers may contribute to the lateral dispersion of migrating ferret neurons within the SVZ. As neurons approach the IZ and enter the CP, the increased parallel nature of the radial fiber scaffold, together with less fiber switching, may produce more radial migration. It should be noted that, although the cortical architecture appeared to be stable during the imaging sessions, the in vivo ferret RG fiber scaffold might differ from that of ex vivo ferret cortical slices used in this study.

The meandering and turning behaviors displayed by migrating ferret neurons suggests a possible modification to the radial unit hypothesis. According to this model, neurons climb radially along one or more neighboring RG fibers so that a spatial map of the $\mathrm{VZ}$ is projected to the $\mathrm{CP}$ in an approximately point-to-point manner (Rakic, 1988; Rakic, 1995). Recent clonal studies of neuronal distribution in the mouse cortex are consistent with this model (Yu et al., 2009). However, retroviral studies revealing the lateral dispersion of clonally related neurons in both the ferret (Reid et al., 1997; Ware et al., 1999) and primate (Kornack and Rakic, 1995) suggest that the model may not be strictly consistent with the development of all species and neuronal migration in the gyrencephalic cortex, in particular, may not be strictly radial. Our real-time data provide evidence that ferret pyramidal neurons do not follow the trajectory of a single parent RG fiber, as many rodent neurons do (Noctor et al., 2001), but instead migrate consecutively along several different vRG and oRG fibers. An observation consistent with this behavior was made many years ago based on electron microscopy images of the developing macaque cortex, where the leading process of a migrating neuron was noted to reach out and contact a neighboring RG fiber (Rakic et al., 1974). Therefore, the horizontal dispersion during neuronal migration reported here for the developing ferret cortex may also occur in other large-brain mammals, including the primate.

Together, our data suggest that the lateral dispersion of upperlayer pyramidal neurons when the ferret cortex has just begun to fold is caused by the migration of neurons along a discontinuous path using a diversity of RG fiber scaffolds. It will be interesting to compare neuronal migration properties across a range of lissencephalic and gyrencephalic species to determine whether these findings are general to species with an expanded outer SVZ and/or folded cortex (Lui et al., 2011). In addition, it will be important to determine whether there is a different degree of horizontal dispersion of excitatory neurons migrating within a developing gyrus versus sulcus. This study supports the use of nonrodent model systems for understanding cellular dynamics relevant to human neuronal migration disorders.

\section{References}

Abbah J, Juliano SL (2014) Altered migratory behavior of interneurons in a model of cortical dysplasia: the influence of elevated GABAA activity. Cereb Cortex 24:2297-2308. CrossRef Medline

Ang ES Jr, Haydar TF, Gluncic V, Rakic P (2003) Four-dimensional migratory coordinates of GABAergic interneurons in the developing mouse cortex. J Neurosci 23:5805-5815. Medline

Betizeau M, Cortay V, Patti D, Pfister S, Gautier E, Bellemin-Ménard A, Afanassieff M, Huissoud C, Douglas RJ, Kennedy H, Dehay C (2013) Precursor diversity and complexity of lineage relationships in the outer subventricular zone of the primate. Neuron 80:442-457. CrossRef Medline

Borrell V, Reillo I (2012) Emerging roles of neural stem cells in cerebral cortex development and evolution. Dev Neurobiol 72:955-971. CrossRef Medline

Borrell V, Kaspar BK, Gage FH, Callaway EM (2006) In vivo evidence for radial migration of neurons by long-distance somal translocation in the developing ferret visual cortex. Cereb Cortex 16:1571-1583. Medline

Butt SJ, Fuccillo M, Nery S, Noctor S, Kriegstein A, Corbin JG, Fishell G (2005) The temporal and spatial origins of cortical interneurons predict their physiological subtype. Neuron 48:591-604. CrossRef Medline

Cobos I, Long JE, Thwin MT, Rubenstein JL (2006) Cellular patterns of transcription factor expression in developing cortical interneurons. Cereb Cortex 16:i82-i88. Medline

Edmondson JC, Hatten ME (1987) Glial-guided granule neuron migration in vitro: a high-resolution time-lapse video microscopic study. J Neurosci 7:1928-1934. Medline

Fietz SA, Kelava I, Vogt J, Wilsch-Bräuninger M, Stenzel D, Fish JL, Corbeil D, Riehn A, Distler W, Nitsch R, Huttner WB (2010) OSVZ progenitors of human and ferret neocortex are epithelial-like and expand by integrin signaling. Nat Neurosci 13:690-699. CrossRef Medline

Fogarty M, Grist M, Gelman D, Marín O, Pachnis V, Kessaris N (2007) Spatial genetic patterning of the embryonic neuroepithelium generates GABAergic interneuron diversity in the adult cortex. J Neurosci 27: 10935-10946. CrossRef Medline

Gertz CC, Lui JH, LaMonica BE, Wang X, Kriegstein AR (2014) Diverse behaviors of outer radial glia in developing ferret and human cortex. J Neurosci 34:2559-2570. CrossRef Medline

Gray SJ, Foti SB, Schwartz JW, Bachaboina L, Taylor-Blake B, Coleman J, Ehlers MD, Zylka MJ, McCown TJ, Samulski RJ (2011) Optimizing promoters for recombinant adeno-associated virus-mediated gene expression in the peripheral and central nervous system using self-complementary vectors. Human gene therapy 22:1143-1153. CrossRef Medline

Hansen DV, Lui JH, Parker PR, Kriegstein AR (2010) Neurogenic radial glia in the outer subventricular zone of human neocortex. Nature 464:554561. CrossRef Medline

Haubensak W, Attardo A, Denk W, Huttner WB (2004) Neurons arise in the basal neuroepithelium of the early mammalian telencephalon: a major site of neurogenesis. Proc Natl Acad Sci U S A 101:3196-3201. CrossRef Medline

Jackson CA, Peduzzi JD, Hickey TL (1989) Visual cortex development in the ferret. I. Genesis and migration of visual cortical neurons. J Neurosci 9:1242-1253. Medline

Kornack DR, Rakic P (1995) Radial and horizontal deployment of clonally related cells in the primate neocortex: relationship to distinct mitotic lineages. Neuron 15:311-321. CrossRef Medline

LaMonica BE, Lui JH, Hansen DV, Kriegstein AR (2013) Mitotic spindle orientation predicts outer radial glial cell generation in human neocortex. Nat Commun 4:1665. CrossRef Medline

Lui JH, Hansen DV, Kriegstein AR (2011) Development and evolution of the human neocortex. Cell 146:18-36. CrossRef Medline

Lukaszewicz A, Savatier P, Cortay V, Giroud P, Huissoud C, Berland M, Kennedy H, Dehay C (2005) G1 phase regulation, area-specific cell cycle control, and cytoarchitectonics in the primate cortex. Neuron 47: 353-364. CrossRef Medline

Malatesta P, Hartfuss E, Götz M (2000) Isolation of radial glial cells by fluorescent-activated cell sorting reveals a neuronal lineage. Development 127:5253-5263. Medline

Marín O, Rubenstein JL (2001) A long, remarkable journey: tangential migration in the telencephalon. Nat Rev Neurosci 2:780-790. Medline

Martínez-Cerdeño V, Cunningham CL, Camacho J, Antczak JL, Prakash AN, Cziep ME, Walker AI, Noctor SC (2012) Comparative analysis of the subventricular zone in rat, ferret and macaque: evidence for an outer subventricular zone in rodents. PLoS One 7:e30178. CrossRef Medline

Martini FJ, Valiente M, López Bendito G, Szabó G, Moya F, Valdeolmillos M, Marín O (2009) Biased selection of leading process branches mediates chemotaxis during tangential neuronal migration. Development 136: 41-50. CrossRef Medline

McConnell SK (1988) Fates of visual cortical neurons in the ferret after isochronic and heterochronic transplantation. J Neurosci 8:945-974. Medline

Miyata T, Kawaguchi A, Okano H, Ogawa M (2001) Asymmetric inheri- 
tance of radial glial fibers by cortical neurons. Neuron 31:727-741. CrossRef Medline

Miyoshi G, Hjerling-Leffler J, Karayannis T, Sousa VH, Butt SJ, Battiste J, Johnson JE, Machold RP, Fishell G (2010) Genetic fate mapping reveals that the caudal ganglionic eminence produces a large and diverse population of superficial cortical interneurons. J Neurosci 30:1582-1594. CrossRef Medline

Nadarajah B, Brunstrom JE, Grutzendler J, Wong RO, Pearlman AL (2001) Two modes of radial migration in early development of the cerebral cortex. Nat Neurosci 4:143-150. CrossRef Medline

Noctor SC, Scholnicoff NJ, Juliano SL (1997) Histogenesis of ferret somatosensory cortex. J Comp Neurol 387:179-193. Medline

Noctor SC, Flint AC, Weissman TA, Dammerman RS, Kriegstein AR (2001) Neurons derived from radial glial cells establish radial units in neocortex. Nature 409:714-720. CrossRef Medline

Noctor SC, Flint AC, Weissman TA, Wong WS, Clinton BK, Kriegstein AR (2002) Dividing precursor cells of the embryonic cortical ventricular zone have morphological and molecular characteristics of radial glia. J Neurosci 22:3161-3173. Medline

Noctor SC, Martínez-Cerdeño V, Ivic L, Kriegstein AR (2004) Cortical neurons arise in symmetric and asymmetric division zones and migrate through specific phases. Nat Neurosci 7:136-144. CrossRef Medline

O’Rourke NA, Dailey ME, Smith SJ, McConnell SK (1992) Diverse migratory pathways in the developing cerebral cortex. Science 258:299-302. CrossRef Medline

O'Rourke NA, Sullivan DP, Kaznowski CE, Jacobs AA, McConnell SK (1995) Tangential migration of neurons in the developing cerebral cortex. Development 121:2165-2176. Medline

O’Rourke NA, Chenn A, McConnell SK (1997) Postmitotic neurons migrate tangentially in the cortical ventricular zone. Development 124: 997-1005. Medline

Ostrem BE, Lui JH, Gertz CC, Kriegstein AR (2014) Control of outer radial glial stem cell mitosis in the human brain. Cell Rep 8:656-664. CrossRef Medline

Poluch S, Juliano SL (2015) Fine-tuning of neurogenesis is essential for the evolutionary expansion of the cerebral cortex. Cereb Cortex 25:346-364. CrossRef Medline

Qin JY, Zhang L, Clift KL, Hulur I, Xiang AP, Ren BZ, Lahn BT (2010) Systematic comparison of constitutive promoters and the doxycyclineinducible promoter. PLoS One 5:e10611. CrossRef Medline

Rakic P (1971) Guidance of neurons migrating to the fetal monkey neocortex. Brain Res 33:471-476. CrossRef Medline

Rakic P (1972) Mode of cell migration to the superficial layers of fetal monkey neocortex. J Comp Neurol 145:61-83. CrossRef Medline

Rakic P (1988) Specification of cerebral cortical areas. Science 241:170-176. CrossRef Medline
Rakic P (1995) A small step for the cell, a giant leap for mankind: a hypothesis of neocortical expansion during evolution. Trends Neurosci 18:383-388. CrossRef Medline

Rakic P, Stensas LJ, Sayre E, Sidman RL (1974) Computer-aided threedimensional reconstruction and quantitative analysis of cells from serial electron microscopic montages of foetal monkey brain. Nature 250:3134. CrossRef Medline

Reid CB, Tavazoie SF, Walsh CA (1997) Clonal dispersion and evidence for asymmetric cell division in ferret cortex. Development 124:2441-2450. Medline

Reillo I, Borrell V (2012) Germinal zones in the developing cerebral cortex of ferret: ontogeny, cell cycle kinetics, and diversity of progenitors. Cereb Cortex 22:2039-2054. CrossRef Medline

Reillo I, de Juan Romero C, García-Cabezas MÁ, Borrell V (2011) A role for intermediate radial glia in the tangential expansion of the mammalian cerebral cortex. Cereb Cortex 21:1674-1694. CrossRef Medline

Smart IH, McSherry GM (1986a) Gyrus formation in the cerebral cortex in the ferret. I. Description of the external changes. J Anat 146:141-152. Medline

Smart IH, McSherry GM (1986b) Gyrus formation in the cerebral cortex of the ferret. II. Description of the internal histological changes. J Anat 147: 27-43. Medline

Smart IH, Dehay C, Giroud P, Berland M, Kennedy H (2002) Unique morphological features of the proliferative zones and postmitotic compartments of the neural epithelium giving rise to striate and extrastriate cortex in the monkey. Cereb Cortex 12:37-53. CrossRef Medline

Tabata H, Nakajima K (2003) Multipolar migration: the third mode of radial neuronal migration in the developing cerebral cortex. J Neurosci 23:9996-10001. Medline

Tabata H, Kanatani S, Nakajima K (2009) Differences of migratory behavior between direct progeny of apical progenitors and basal progenitors in the developing cerebral cortex. Cereb Cortex 19:2092-2105. CrossRef Medline

Takahashi T, Nowakowski RS, Caviness VS Jr (1996) Interkinetic and migratory behavior of a cohort of neocortical neurons arising in the early embryonic murine cerebral wall. J Neurosci 16:5762-5776. Medline

Valiente M, Martini FJ (2009) Migration of cortical interneurons relies on branched leading process dynamics. Cell Adhesion Migration 3:278-280. CrossRef Medline

Ware ML, Tavazoie SF, Reid CB, Walsh CA (1999) Coexistence of widespread clones and large radial clones in early embryonic ferret cortex. Cereb Cortex 9:636-645. CrossRef Medline

Yu YC, Bultje RS, Wang X, Shi SH (2009) Specific synapses develop preferentially among sister excitatory neurons in the neocortex. Nature 458: 501-504. CrossRef Medline 